\title{
Exploring Canons \& Cathedrals with Open Virtual Worlds The Recreation of St Andrews Cathedral, St Andrews Day, 1318
}

\author{
S Kennedy*, R Fawcett ${ }^{\dagger}$, A Miller*, L Dow*, R Sweetman ${ }^{\ddagger}$, A Field*, A Campbell*, I Oliver* J. McCaffery* and C Allison* \\ ${ }^{*}$ School of Computer Science, University of St Andrews Email: see http://www.cs.st-andrews.ac.uk/directory ${ }^{\dagger}$ \\ ${ }^{\dagger}$ School of Art History, University of St Andrews Email: rf41@ st-andrews.ac.uk ${ }^{\dagger}$, \\ ${ }^{\ddagger}$ School of Classics, University of St Andrews Email: rs43@st-andrews.ac.uk ${ }^{\ddagger}$,
}

\begin{abstract}
St Andrews Cathedral is located on the East Coast of Scotland. Construction started in 1160 and spanned Romanesque and Gothic architectural styles. It was consecrated in 1318, four years after the battle of Bannockburn in the presence of King Robert I. For several hundred years, the Cathedral was one of the most important religious buildings in Europe and the centre of religious life in Scotland. During the Reformation, John Knox himself lead reformers in divesting the Cathedral of all its finery. Thereafter it fell into disuse and decline. Today the remains hint at its former glory. Here the use of Open Virtual Worlds (OVW) to support new modes of engagement with cultural heritage is presented through the example of St Andrews Cathedral.
\end{abstract}

Open Virtual Worlds offer an extensible collaborative environment for developing historical scenes against which background material and intangible aspects of cultural heritage associated with a site may be explored. They offer the potential to reconstruct within a 3D computer environment both the physical structures of the past and important aspects of the lighting, sounds and lifestyles that once existed within those structures. Bringing together architecture, sculpture, illumination, stained-glass, music, procession and lighting into a scene, which can be explored from multiple spatial perspectives enables holistic appreciations to be developed.

\section{INTRODUCTION}

For over four hundred years St Andrews Cathedral stood at the centre of Scotland's religious and political life, yet today there are insufficient remains for the spectacle of the building in its prime to be obvious. Work on creating a digital recreation started in 2009 as a collaboration between computer scientists, archaeologists and historians. The goal was to develop a 3D model of the Cathedral informed by physical evidence, scholarship and expert interpretation. In so doing appreciation of the Cathedral's form previously confined to the minds of scholars would become accessible to new audiences.

The significance of the Cathedral lies not only in the stonework but also the function that it performed, the people who lived in it and history that it was part of. The OVW environment the model is located within (Open Simulator) enables the modelling of both material culture: the surrounding landscape, architecture, fixtures and paintings and intangible culture: the interplay of light, weather and architecture; sounds of chant and the routines of the Opus Dei, hence virtual visitors experience medieval religious life.

A virtual visitor is represented in world by the proxy of an Avatar, which they control much as a user would in a computer game having a choice of both first (Call of Duty and Crysis) and third person (Tomb Raider and Resident Evil) perspectives. Users control where the avatar goes, looks and interacts using interfaces and digital literacies developed playing games like Call of Duty, thereby making cultural heritage accessible in powerful new ways.

The environment is fully programmable enabling visitors to participate in historic scenes whilst hearing the stories of historic characters they meet such as Bishop William de Lamberton, King Robert I, the Cannons of the Cathedral and visiting pilgrims. The model itself provides a 3D interface to the Web; text, video and social media resources, which are accessed through clicking on

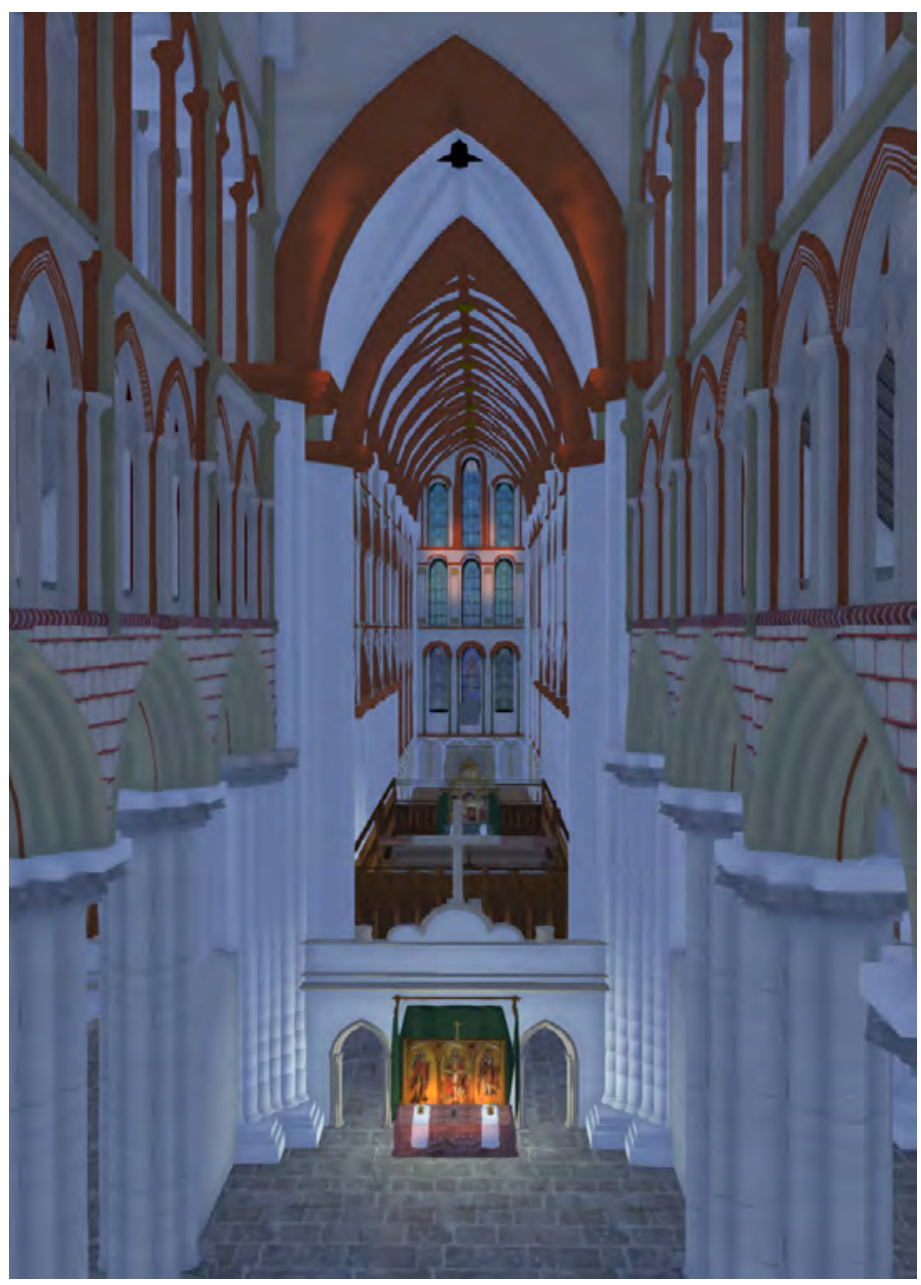

Fig. 1. Internal view towards Choir St Andrews Day 1318.

hotspots. Thus the reconstruction introduces the visitor to wider historic narratives and acts as a 3D interface to relevant web resources.

The reconstruction has been deployed in diverse scenarios. It is accessible as a free live Internet service that users connect to worldwide (openvirtualworlds.org/start); as an installation located in a museum with panoramic screen, natural movement control and immersive sound; and as a portable multi user exhibition, which has been used in schools, science centres, festivals and museums. This flexibility of deployment makes an important contribution to Cultural Heritage [1], by offering the potential of mass participation in the creation and propagation of cultural heritage.

The paper is organised into the following four sections: the site and subject of the recreation, the OVW technology used, the methodology applied and real world scenarios where the reconstructions have been deployed and used are described. 


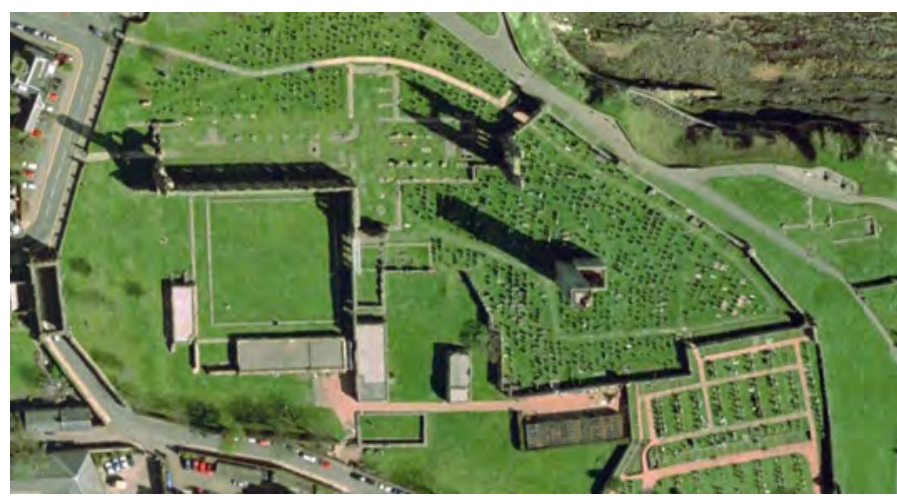

Fig. 2. Arial View of Cathedral and Cloister remains in grounds 2011

II. St Andrews Cathedral Site:

NARRATIVES FROM INDEPENDENCE TO THE REFORMATION

St Andrews Cathedral occupies a site used for worship since the $8^{\text {th }}$ Century AD. Work on the Cathedral began around 1160 and was completed nearly 150 years later (the west faade and parts of the nave collapsed in a storm around 1270). It was finally consecrated in 1318 four years after the battle of Bannockburn and in the presence of King Robert I of Scotland. St Andrews Cathedral was in its prime, the centre of Scotland's religious life, its largest and most magnificent church. In 1378 the Cathedral suffered a significant fire prompting a reworking of many of its features including the West and East End windows. Its presence was the catalyst for the foundation of a university at St Andrews in the early fifteenth century [2], which remains an important seat of learning to this day. In 1561 following the Scottish reformation the Cathedral was abandoned by the Bishops and replaced by the parish church as the chief place of worship. The former headquarters of the Scottish Church was left to fall into ruin, with much of its stone being used in the construction of town dwellings.

During its time the Cathedral was central to Scottish personalities and history. St Andrews was the highest ranking Scottish see. The establishment of Augustinian Cannons followed by the initiation of building work by Bishop Ernald reflected integration with the European church, economic dynamism and decline of the Celtic Church. The diocese funded Robert Bruce during the Wars of Independence. Its Bishop William de Lamberton contributed to the formulation of the Declaration of Arbroath, a central document in the formation of Scottish Nationhood. Isabella, sister of Donnchadh IV, last Pictish Earl of Fife, crowned Bruce King. John Knox personally lead his congregation against the Cathedral's finery and following the murder of Cardinal Beaton the first Scottish protestant congregation was established in the Bishop's palace.

The date chosen for the reconstruction was 1318, the year of its consecration. Important fragments of the remain. The east gable of the presbytery, where the relics of St Andrew were purported to be kept, along with the south wall of the nave, and the majestic West Entrance all point to the Cathedral's former majesty. The cloister retains its ruined chapter house and stone-vaulted under crofts. Consequently, much evidence of the Cathedral's form exists. An aerial view of the current monument is shown in fig. 2 and a view from the nave looking towards the choir in fig. 3.

The reconstruction process involved collaboration between computer scientists, archaeologists and art historians. It drew upon existing resources relating to the Cathedral: surviving architecture on site,

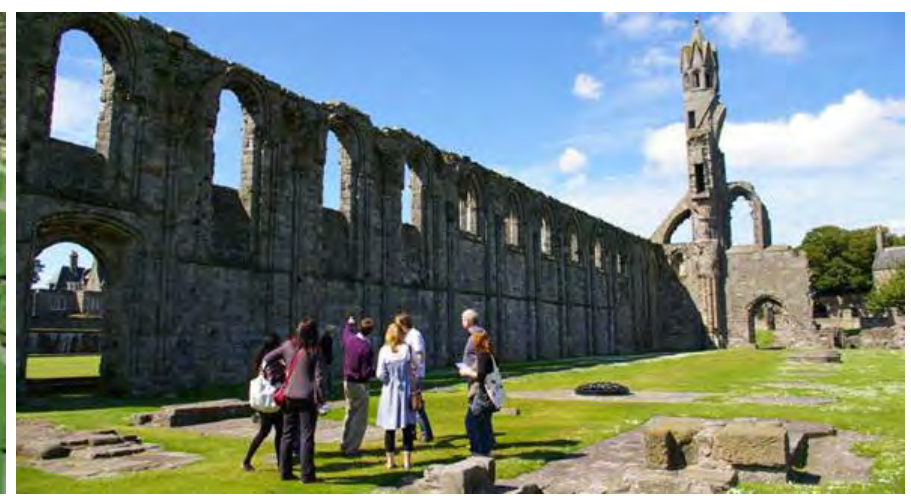

Fig. 3. Site visit showing View from Knave to Choir

architectural fragments both in the museum and reused throughout the town, the pre existing work of medieval architectural historians [3], [2] along with architectural drawings and artistic impressions of the Cathedral's original appearance. These resources were drawn upon to re-construct the Cathedral and surrounding buildings including the earlier church of St Regulus.

\section{A PLATFORM FOR VIRTUAL RECREATION}

Virtual worlds are distributed 3D systems which enable users to create and edit their own content whilst the system is live. The user interfaces, both for navigation and modelling are intuitive and easy to use. This enables domain experts and technical people to collaborate together in the creation of historic scenes.

Open Simulator (OpenSim) is a freely available OVW environment which can be used to develop [4] and deliver historical reconstructions [5] and serious games. It is distributed under a creative commons attribution licence, which gives considerable flexibility to extend its functionality and to deploy it in different scenarios. OpenSim is an application server, which integrates with the World Wide Web. At the heart of an OpenSim application is an extensible, mutable, 3D environment users inhabit through the proxy of an avatar. Other users are able to see the avatar's actions and engage in synchronous communication through movement, gestures, text chat, and voice. This projection of presence enables both collaborative exploration.

An OpenSim OVW is made up of three discrete components: the client, the simulations and services.

Simulations may model the weather, the physical structure of a space, the actions of avatars and interactions with other Internet resources. The amount, colour and movement of clouds, the colour opacity and reflectivity of water, visibility and mist, lighting and the presence of shadows as well as the time of day are all configurable. The surface of the region may be defined using external real world data and textures applied to give the impression of grassy plains, rocky hills, deserts or forests. The structure of the architecture, fittings and furnishings of buildings may be constructed in-world out of primitives (prims) or imported as meshes.

The movement and appearance of avatars is customizable. Inworld tools provide control over body shape, size and colouring as well as the garments that are worn. More control is achieved by using external modelling programs to design clothing, body shapes and animations. Libraries of clothes, body shapes and animations may be freely imported and exported. Avatars may also represent in world Non Player Characters (NPC), that have preprogrammed responses 


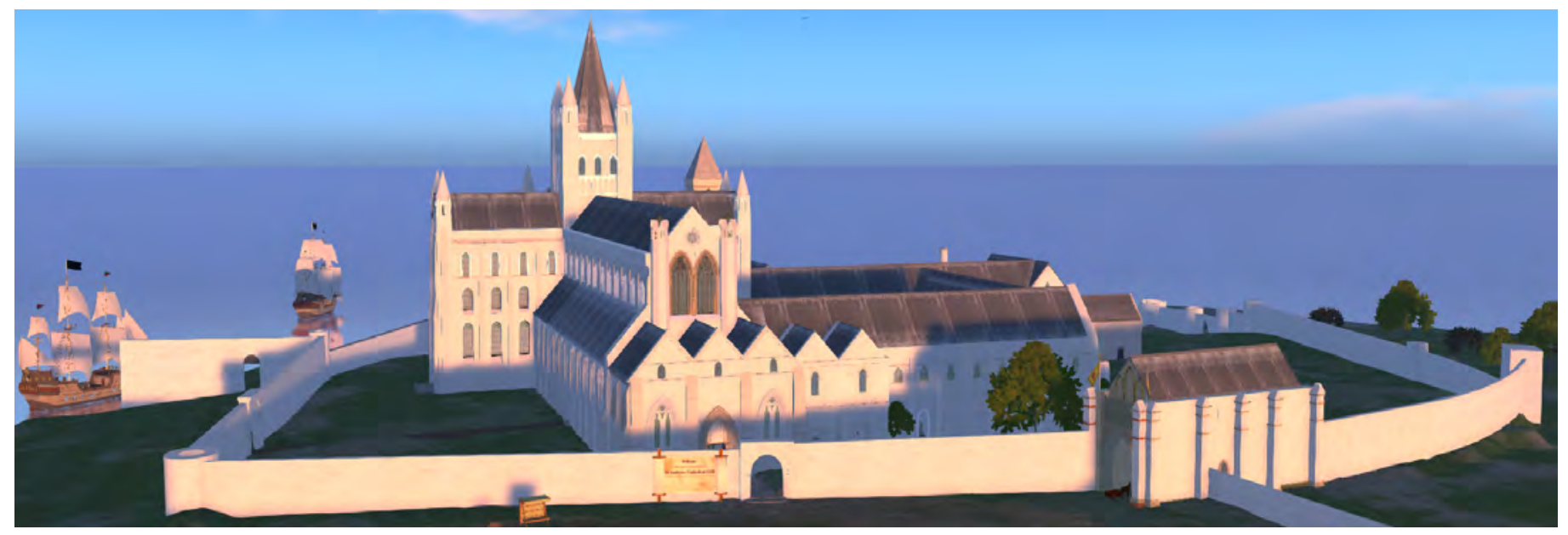

Fig. 4. St Andrews Cathedral recreation set on the sunny St Andrews day afternoon 1318, showing the West Gate in the foreground and ships in the background.

to events and artificial intelligence. The libopenmetaverse [6] library provides support for creating avatar bots controlled remotely by a programmable client.

Soundscapes are created by streaming to virtual locations, clips may be embedded in objects and played in response to events. Voice over IP is used for peer to peer voice chat. Media textures may be attached to prims allowing media to be streamed and displayed on the Prim surface. Web pages can also be embedded on a surface and set to point at a web address. Web pages may be loaded into a browser when events trigger scripts embedded in objects. OpenSim is written in $\mathrm{C \#}$ and runs over the .net framework or mono. In world scripting is supported in LSL and C\#, this allows Avatars or other objects to respond to events, such as proximity, being touched or receiving messages on a chat channel. Regions modules allow for powerful OVW applications to be created programmatically [7].

The server architecture supports multiple deployment scenarios including: mobile access, installations, institutional services and Internet access. The user controls the environment through keyboard and mouse, gestures, games controllers, or touch screens. Scenes are viewed in stereoscopic 3D through an Oculus Rift headset, on a tablet, on a high definition screen or on a synchronised multi projection surface as part of an installation. The flexible architecture and multiple control modes mean many use cases are supported.

Support for modelling terrain architecture, sculptures and furnishings, as well as intangible elements of cultural heritage such as music and the interactions between people make OVWs an attractive platform for multi-faceted recreations of historic scenes that allow individual pieces of culture to be placed within context and understood holistically.

\section{RECREATING THE CATHEDRAL'S FORMER MAJESTY.}

The virtual St Andrews Cathedral was envisaged originally as a tool for learning in Schools and Universities. As such its has been used as part of programs of study complementing traditional scholarship and visits to the physical site. This work goes beyond 3D modelling and reconstruction to aim at the recreation of an historic scene, we attempt Virtual Recreation as defined by the Seville Charter [8].

Virtual Recreation: this involves using a virtual model to visually recover an archaeological site at a given moment in the past, including material culture (movable and immovable heritage), environment, landscape, customs, and general cultural significance.

At the heart of the recreation process lies collaboration between experts in the archaeology and history of the monument, graphical designers capable of developing 3D models which match the vision of the historians and computer scientists who develop delivery systems. The OVW provides an environment which facilitates such collaboration. Iterations of the model can be viewed and evaluated by domain experts who in turn give direction to developers.

A number of discrete but interconnected steps make up the process of reconstruction: research, setting the scene, landscaping, architecture, furnishings and fittings, soundscape, character development, linkages to web and social media as well as the development scenarios.

The reconstruction draws upon existing scholarship in medieval religious buildings, medieval books and medieval music. Contextualisation of surviving architecture on the site and reference to surviving features on other sites inform the reconstruction. Research into personalities contemporaneous with the Cathedral and modes of dress inform the creation of in world characters.

Real world ordnance survey point heights provided the framework for the terrain. The OS coordinates for the monument were identified; the point heights were then scaled to minimize quantization error and loaded into a bit map image file. The map was then transformed using cubic interpolation from one point per 10 meter to one point per meter. The image map was then loaded into the OpenSim region. The in world height and the scale of the land were then adjusted to agree with the real world heights.

The process of reconstructing the architecture was divided into three substages: establishing scale, layout and orientation; developing external form; and establishing the internal form and features. Each was iterative. Initial discussions identified evidence to work from and a plan for development, regular meetings between developers and historians were held. During the meetings virtual tours of the virtual building site were conducted and discussions held as to the correctness and appropriateness of the current content.

The first stage was to create or locate scale architectural drawings of floor plans, elevations and details. The floor plans were imported as an image, laid on the ground and scaled to provide a blue print 

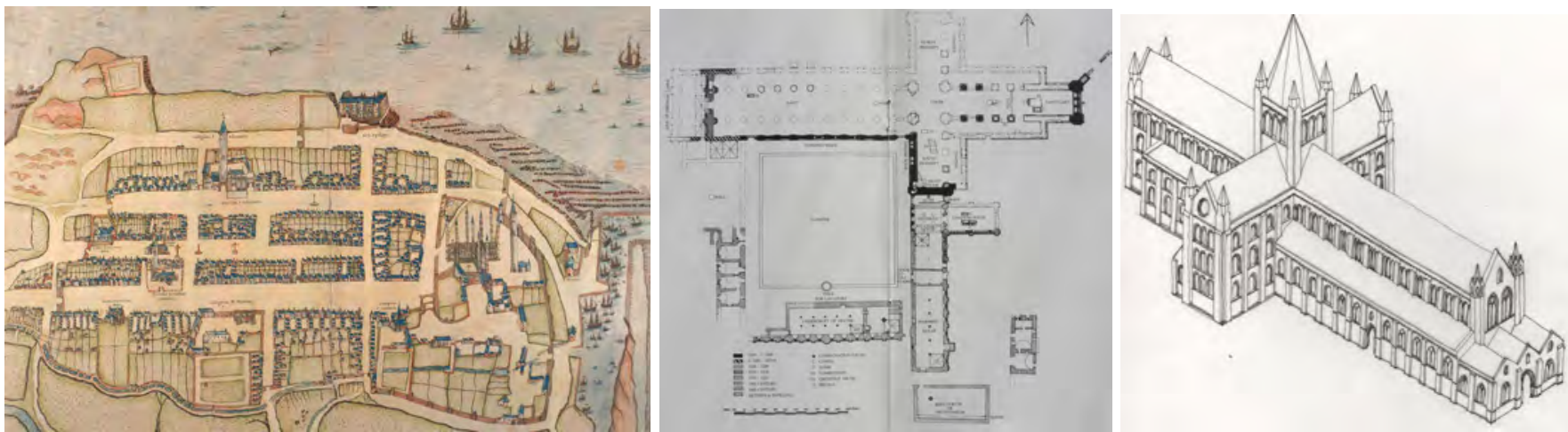

Fig. 5. The Geddy view of St Andrews

Fig. 6. Scale Floor Plan

Fig. 7. 2D Sketch
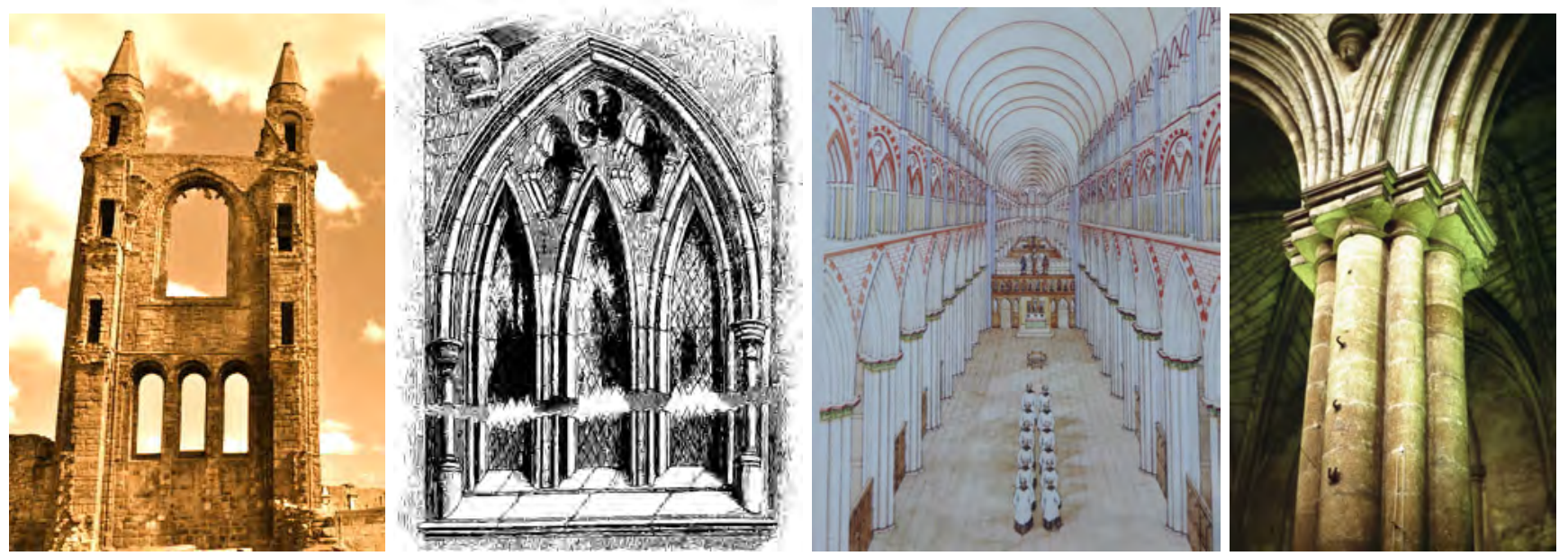

Fig. 8. The East End

Fig. 9. Gothic Window

Fig. 10. Procession

Fig. 11. Ripon
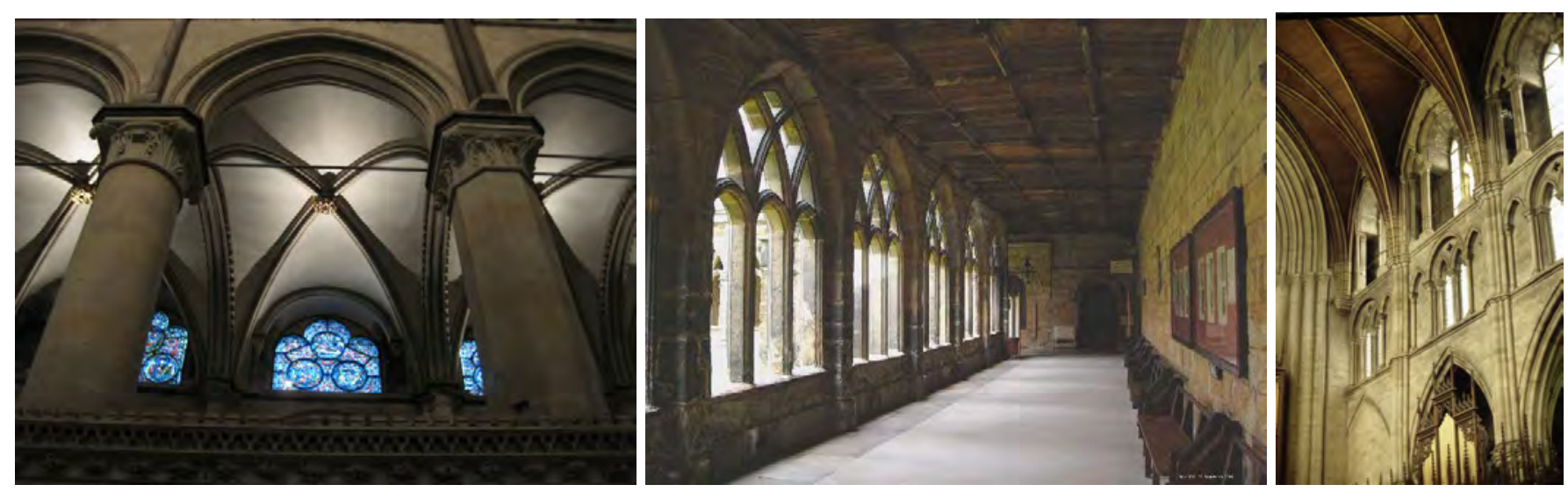

Fig. 12. Nave Vaulting Cantebury

Fig. 13. Cloister in Durham

Fig. 14. Choir

for the location of walls, doors and other features. Primitives were created and shaped to the appropriate size for walls and floors, with spaces being left for windows, doors and other features. Appropriate textures are applied to give an authentic look.

The Geddy view, drawn in 1540 (fig. 5) provides context. The church was laid out in a cross with the length running from East to the West. A scale plan of the Cathedral (fig. 6), a site plan, reconstruction sketches (fig. 7), site photographs (fig. 2) and observations (fig. 3) provided evidence for the reconstruction.

Issues addressed in establishing the external form of the building include the structure of the East and West ends, the form of the win- dows along the length of the choir and nave and the structure of the cloister area. Reconstruction work was based on existing drawings, themselves based on the evaluation of on site physical evidence and reference to surviving features in churches developed in the same architectural style. A series of two dimensional elevations guided proportions. Reference was made to sketches of Gothic window tracery (fig. 9), buttresses and to surviving features in Jedburgh, Elgin and Glasgow Cathedrals.

A photograph of the remaining East End is shown in fig. 16. This shows a lower tier of three windows with a large upper window. This configuration was established after the fire of 1378. Previously, 
there were three tiers as shown in fig. 18. During the construction of the Cathedral architectural tastes changed from Romanesque to Gothic. This is reflected in the form of the nave windows. Towards the west these have simple rounded arches, towards the east the arch is pointed and the windows contain a delicate $\mathrm{Y}$ shaped tracery as shown in fig. 17.

The West End was the ceremonial entrance to the Cathedral used only on special occasions. It consists of a 'magnificent doorway framed by five receding orders of arches, is flanked by tall polygonal turrets.... A stone-vaulted porch in front of the doorway (now gone) stretched across the west front' [9]. Placement and size of the windows in the reconstructed West entrance went through several iterations before arriving at a configuration which reconciled surviving architecture, correct styling and placement in relation to the surviving west port vestibule. An external view is shown in fig. 4 .

The outline of the cloister is clearly discernible from above in fig. 2. The surviving cloister at Durham (fig. 13) provided a model we worked from. The known position of the Cathedral windows provided a guide for the height of the cloister. Located in the cloister is a well. The canons eating (fig. 24) and sleeping quarters (fig. 22), the kitchens (fig. 21) and the Chapter house (fig. 23) can all be accessed from the cloisters. A view from the cloister garden is shown in fig. 17.

The internal architectural features were developed from evaluating existing 2D reconstruction drawings for example fig. 7, surveying surviving features at the site and with reference to architecture at Canterbury (fig. 12), Jedburgh, Ripon Minster (fig. 11) and Durham Cathedral's cloisters (fig. 13).

In recreating the pillars; the base, the columns and how the finial connects the column to vaulting were all considered. The bases of the transept Pillars survive in place and there are surviving pieces of pillars and bases in the Cathedral museum. The pillars at the Cathedral in Ripon (fig. 11) provide a good model for how St Andrews' pillars would have looked. The pillars in the transept, choir and knave differ in style and detail. The Choir pillars are more decorative than the nave reflecting the higher prestige associated with access to these parts.

The vaulting was in the Gothic style, similar to that in the nave at Canterbury Cathedral (fig. 12). Technically it was difficult to model this as the shapes are difficult to arrive at from OpenSim primitives. Consequently, they were created in a 3D modelling program (Blender) and imported.

The choir section contains a tri-foil of surrounding arches as does the choir at Ripon fig. 11. The choir screens played both a decorative and functional role. The relics of St Andrews were purported to be located within the reliquary to the east of the choir. Heights of windows, positioning of the screen and the magnificent vaulted ceiling are all features of this part of the building, captured in the reconstruction fig. 18.

The location of the stairs to the refectory was investigated as the exact position was not clear from surviving architecture. The position adopted makes the most sense in providing access to the well, cloister, kitchen and refectory. The view from the night stair into the transept is shown in fig. 19.

Putting Books back in the Cathedral: Books and manuscripts would have been central to monastery life. The book presses are at the junction between the Cathedral and cloisters. By 1400 the Cathedral had a library of some 100 books. This project has created a model of a Scriptorium, an in-world exhibition of books in the Cathedral and has placed models of appropriate books in the Scriptorium, book presses and lecterns in the Choir, dining areas and Chapter house. In the exhibition visitors can learn about how books were created in the middle ages, gain access to digital copies of rare and valuable books and charters and interact with models of books that were held in the Cathedral's library. They are able to view a videos about book making and to read digitisations of three of the Cathedral's books.

Soundscape: Sound is streamed into the virtual cathedral to provide a realistic audio backdrop. The University of St Andrews choir recorded plainsong chants appropriate for an Augustinian house of that period. With a microphone and headphones visitors may communicate with each other through voice chat (facilitated by a Freeswitch IP telephony server) even when in different physical locations. Specific sound clips are embedded in the environment to be triggered by appropriate avatar actions. For example a door creaks when opened, a NPC answers a question and the fountain bubbles. Music, commentaries and explanations may be accessed through an embedded web interface. Visitors to the Cathedral are able to hear a soundtrack of music appropriate to the time and place.

\section{A. Characters and Scenes}

Non Player Characters (NPCs) appear as avatars and may be programmed to follow scripted behaviour (figs. 29, 28) and to respond to questions. Scripts are placed within the characters, which enable them to react to events. For example upon being approached by an avatar they greet them by name, respond to questions and follow routine tasks. For St Andrews Cathedral models of King Robert I, Bishop William de Lamberton, Prior John De Forfar, Augustinian Canons, pilgrims and peasants have been developed. For each character, appropriate physical models, clothes and accessories have been researched, designed and created. The characters each have a background and a story to tell.

The characters follow a timetable of events. For example, at 8:00am, there is Morrow Mass. At that time, all of the NPCs representing the Augustinian Canons change into suitable clothing, navigate to suitable locations, and are then animated into realistic stances suitable to their individual character during the mass. By following timetables of such events based upon the Monastic Horarium according to the Regularis Concordia, NPCs within the Cathedral add to the tangibility of the reconstruction by providing realistic actions and movements of the characters that would have been present during everyday life in the Cathedral.

Many of the events are controlled through the use of a graph that is overlaid within the Cathedral, representing both navigational way points and strategic positions throughout the Cathedral, as shown in fig. 29. Normally invisible the paths can be made visible for editing. The graph greatly simplifies the process of scripting the movement of NPCs around the Cathedral, by making use of Dijkstras routing algorithm as a way of finding short paths to target positions. This allows NPCs to navigate by being told where to be rather than how to get there. The graph can be fully edited from within the Cathedral itself allowing for the quick and accurate mapping out of way-points.

NPCs follow simple scripts that allow them to hold conversations with users, responding in both a textual and audible format. These scripts work on a keyword basis, allowing sentences about certain topics to trigger an automated response. The user chooses responses which traverse a predefined conversation tree. They can follow the path that they are most interested in. This allows for varied conversations whilst enabling detailing responses and keeps script authoring simple. 

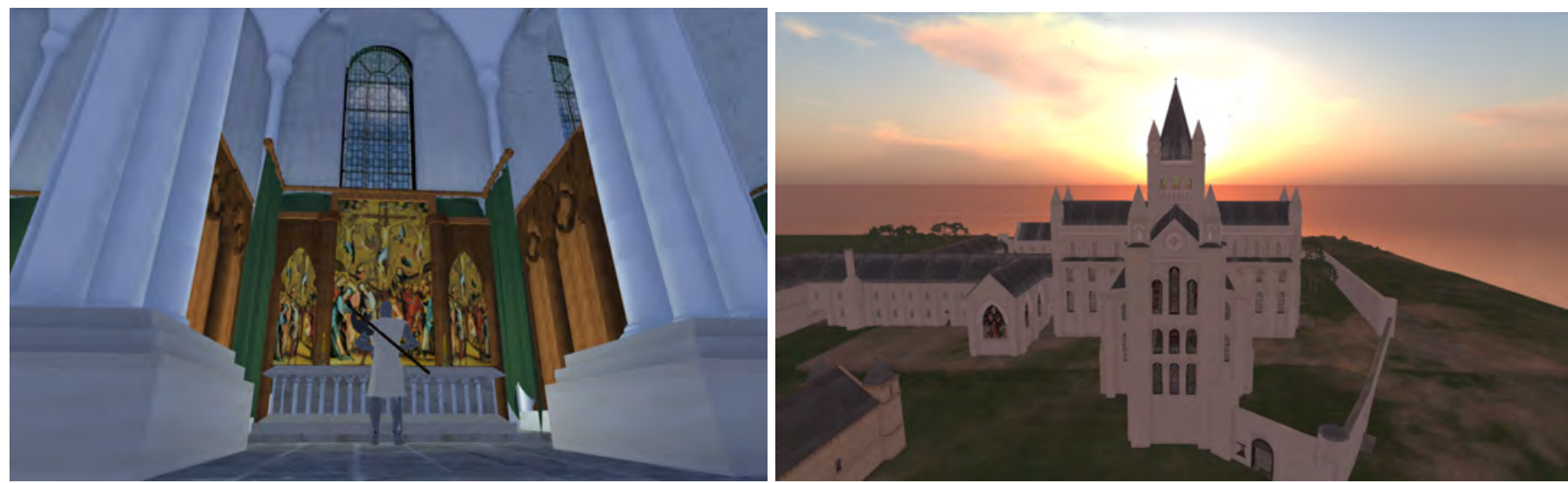

Fig. 15. Robert Bruce in St Andrews Transepts
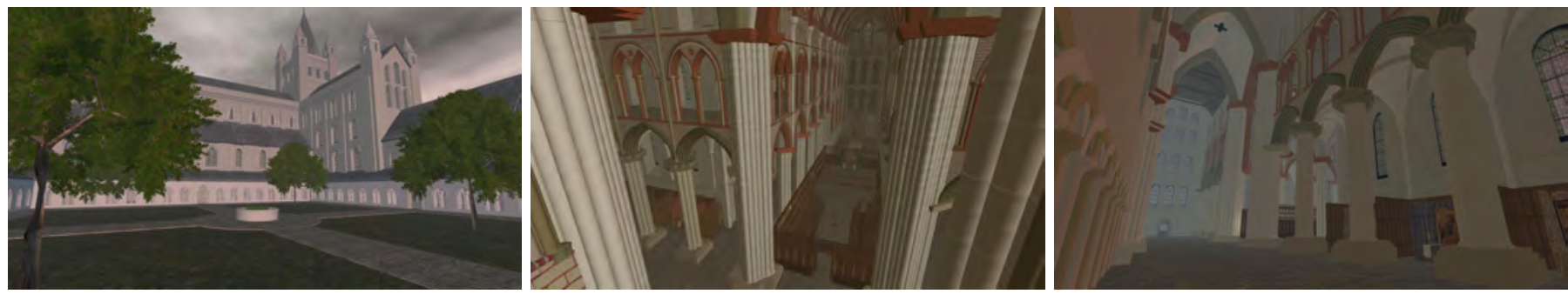

Fig. 17. Cathedral Cloisters

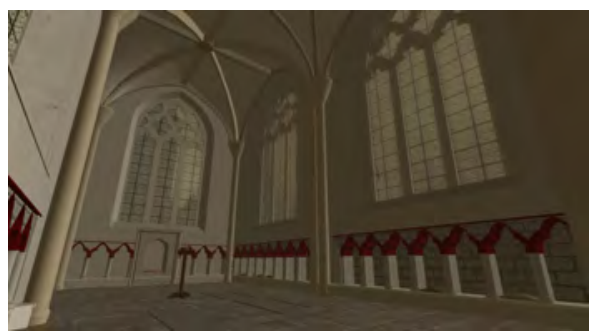

Fig. 20. The Chapter House

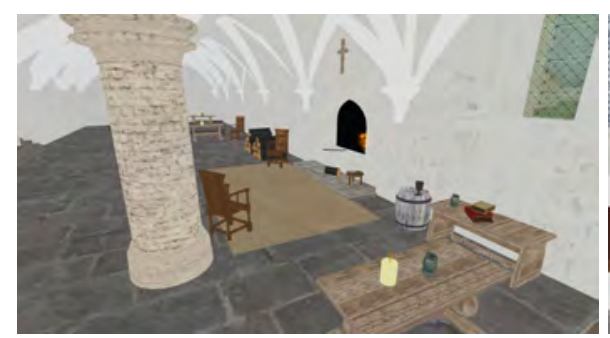

Fig. 23. Warming house
Fig. 18. Internal view to East

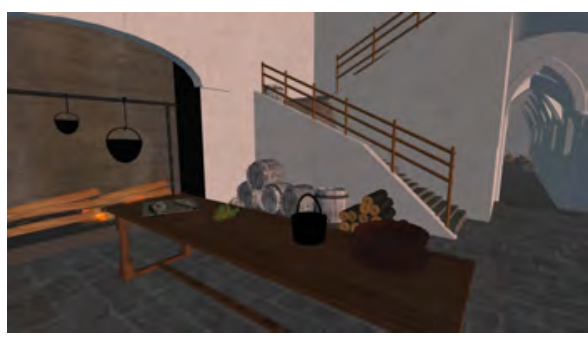

Fig. 21. Cathedral Kitchens

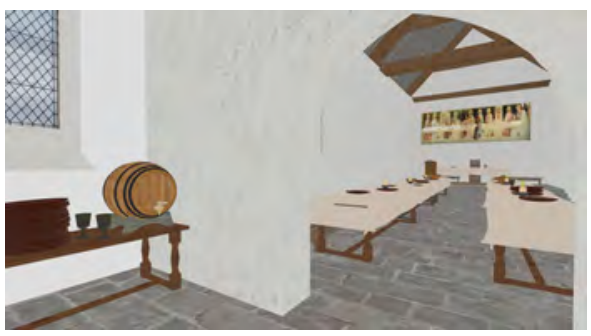

Fig. 24. Cloister dining area
Fig. 19. Transept from night stair

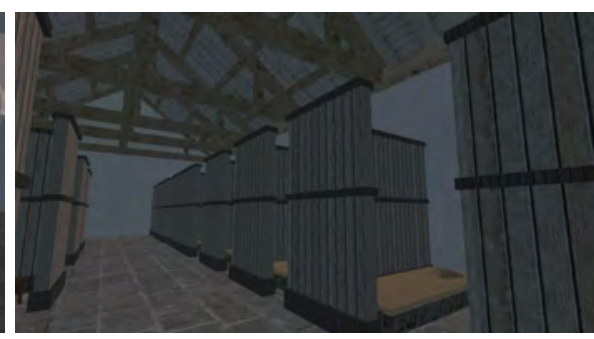

Fig. 22. Sleeping quarters

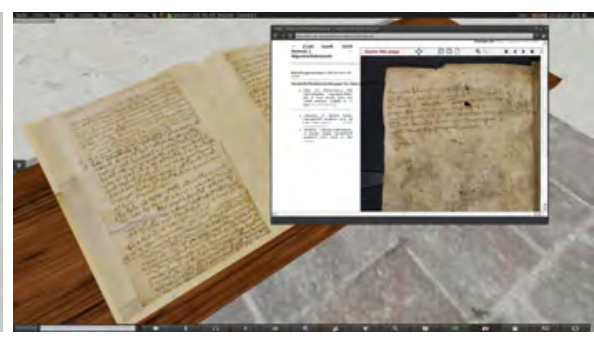

Fig. 25. Book Exhibition

\section{B. Summary and Reflections on the Virtual Recreation process}

The recreation is based upon the historic record. Evidence from remaining architecture, established scholarship and informed interpretation are used. The recreation process used was iterative and development guided by domain experts, resulting in an authentic reconstruction and transparent design process; as documented in this paper. The process of virtual recreation has itself been enlightening:

The ability to walk around the building whilst under construction and to view the model from a multitude of viewpoints allows the placement of features that are missing from the archaeological record to be explored and tested from the perspective of spacial dynamics. This is particu- larly relevant to the placement of doors, the direction of stairways and establishing the function of rooms. These aspects can be considered in an holistic way adding insights gleaned from the reconstruction to the existing knowledge base. Dr Rebecca Sweetman, Classics, University of St Andrews

From the historians perspective the reconstruction of the Cathedral involves both the mental reconstruction of modified and lost features, and the establishment of the range of ways in which buildings that represent a spirituality alien to modern times were intended to function. As such it offers an invaluable academic discipline for those involved in 

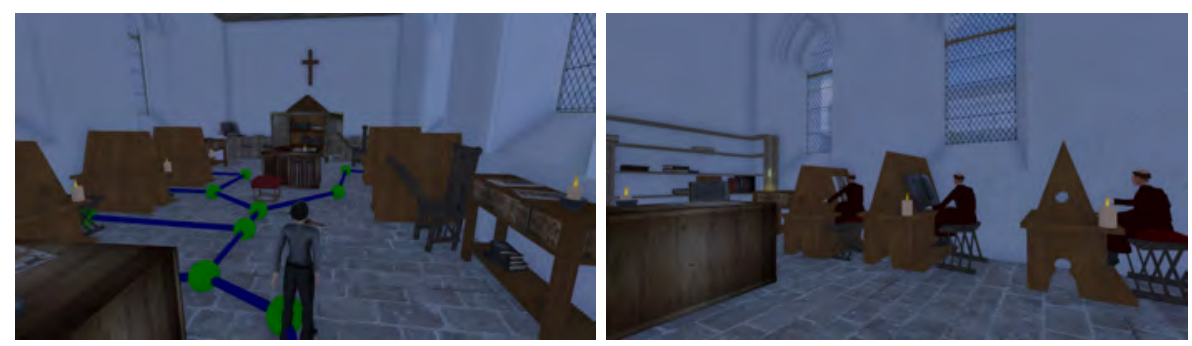

Fig. 26. Editing pathways in scriptorium

Fig. 27. Monks at work in scriptorium

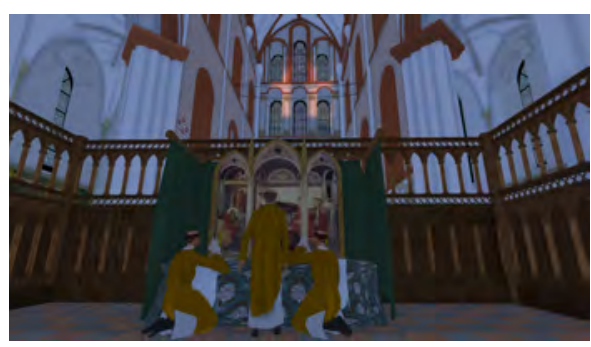

Fig. 28. Morrow mass in the Choir the reconstruction, providing eminently practical ways of testing theories and assumptions. It is then of the greatest value for conveying more widely the understanding that has been gained. Prof. Richard Fawcett, Art History, University of St Andrews

\section{USE CONTEXTS AND EVALUATION}

Over the last few years we have gained experience of using the Cathedral reconstruction in several different contexts. The reconstruction started as a summer project for several Masters students. It has been used as part of degree accredited university courses for undergraduate history and classics courses, in a local unit of the curriculum for year 11 students in a local Secondary school, for all students in a local primary school, as part of a public science exhibition at Dundee Science centre and public events at St Andrews University. There has also been TV and press coverage enabling images of the reconstruction to reach wider audiences.

The Cathedral is hosted on the Apollo OVW server and is accessible over the Internet through a Virtual World client. The Apollo server contains several other reconstructions including Linlithgow Palace, a pre clearance Highland village, 16th century Salt Pans and a sixth century Greek Basilica. A second instantiation is on the OS GRID.

There are circumstances when it is not possible to access the Internet, for example when doing an exhibition in a remote location or in a school. However, using OpenSim this can be overcome by running a local server and connecting clients to it. To enable outreach work we have created a portable exhibition which can be set up in a public space for workshops and classes. This consists of five public facing clients in small form factor PCs equiped with NVidia 680 GPUs and HD 1080p displays, i5 Ivybridge processors and 8 GB of RAM. The server is an i7 with 16GB of RAM and can run 30 regions. The user interface utilized $\mathrm{X}$ - Box controllers along with keyboards and mice.

A terminal was used for monitoring performance and controlling the server. During sessions network traffic traces were captured, along with application load inferred from server frame times and frames per second, traces of memory usage. Connection to the server via WiFi was supported. Several laptops were used to run in world characters that would interact with visitors' avatars along with a tablet demoing a touch screen interface. Several historic characters were created, these included: Bishop William de Lamberton, King Robert the Bruce, an Augustinian Cannon and the Old Grey Lady a ghost reported to haunt the Cathedral.

The reconstruction of St Andrews Cathedral was part of the Sensation exhibition at Dundee Science Centre over the weekend of 16th, 17th and 18th of March 2012. The exhibition was well attended by both local schools and the general public. On Friday several groups of students from local primary schools visited and during Saturday and Sunday there were over 800 visitors representing a wide range of ages and backgrounds. Teachers and pupils who attended this Create and Inspire event expressed an interest in using it in their own schools.

Activities included: free form site exploration; a guided tour by a virtual character; a guided tour by a real world person; a treasure trail/task oriented quest; and playing games of tag or hide and seek against the backdrop of the Cathedral. The experience was evaluated in three ways: use of the system was passvily observed, a visitors book was provided for free form feedback and some questionnaires were distributed.

It was clear throughout that people were attracted to the exhibit and found it easy to use, engaging and educational. All ages were able to quickly master the user interface and navigate the environment with a few seconds instruction. School children were particularly adept at using the game controllers The feedback received was strongly positive. Some forty comments were left in the visitors book a representative sample is given below:

male primary school student: the best place I have ever been love everything inside and will be looking forward to coming back 3 female primary school students: very detailed and interesting. good game. we like Robert the Bruce :) parent: kids were very interested and enjoyed being able to interact with the Cathedral, the controllers were a medium that made it easy for them to do this family group: Fantastic work. Can't wait to visit St Andrews again to look at the Cathedral ruins STEM ambassador: Very interesting would be great to be shown in schools

Over three days hundreds of people interacted with the model. They all took something positive from the experience, whether they were a university lecturer or a primary school student. Embedding the reconstruction within an OVW created a new perspective on Scottish history and made it accessible across the generations. Many expressed a desire to follow up the experience through visiting the ruins or to connect from home to explore in greater detail.

A second example was at a local secondary school as part of the social studies local unit of the Curriculum for Excellence. The unit encouraged students to investigate local history. Activities included an initial talk about archaeology and the history of the Cathedral, a session with the virtual Cathedral, two visits to the Cathedral and a final in class session with a box of relevant artefacts. The students created a portfolio of materials, including drawings, pilgrim's badges and written accounts of imagined pilgrimages to the Cathedral.

The portable exhibition was set up in the school library for three days. Individual sessions were run for classes and it was open for students to use during breaks and at lunchtime. During the classes groups of 4-6 students sat around each terminal. Each group had a 

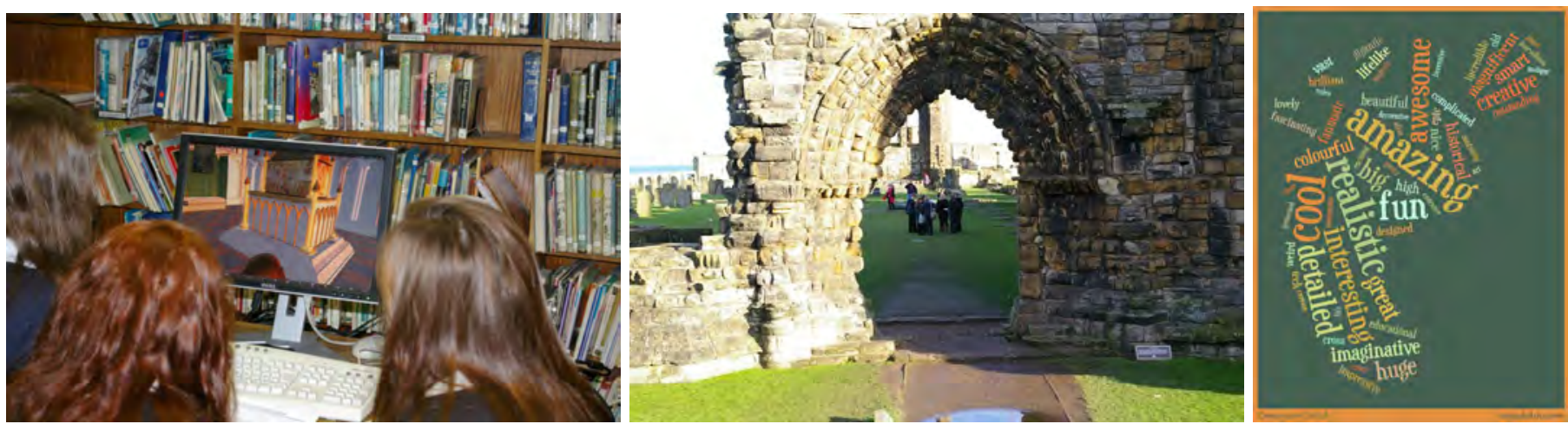

Fig. 29. Exploring the Reliquary from the school library.

Fig. 30. Modern "pilgrims" in the Nave during school visit. Fig. 31. Descriptions.

quest to complete which involved finding different locations within the Cathedral and talking to an avatar representing Robert the Bruce.

On entering the room there was palpable excitement at the prospect of the coming session. The majority of students came equipped with digital literacies that enabled them to use the $\mathrm{x}$-box controllers to explore the Cathedral reconstruction. The few students who did not have experience with $\mathrm{x}$-box controllers were able to quickly learn how to explore using the avatar often with help from their peers.

Having a group of people sharing a work station to achieve a common task meant that they had to co-operate together and negotiate how to share control. This was valuable in developing team working skills. Because the students were using a shared server, they were able to see and interact with other groups' avatars. This added to excitement and engagement with the activity. Interacting with Robert the Bruce was a second highlight of the event. Through following the quest, students were able to learn about the physicality of the Cathedral, to gain a respect for the achievement of such a magnificent building existing 700 years ago and reflect on what it was like to visit the Cathedral in its prime.

The quest had an important role in providing structure to the exploration. Although there was a tendency to become distracted from this, for example flying to explore the edges of the simulation rather than the Cathedral or engaging in irrelevant chat, appropriate supervision addressed this and refocussed the students. The critical factor in the success of the educational experience was the integration of exploration of the model with a wider educational agenda. It helped stimulate investigation and motivate engagement in other activities. Students were able to make connections between exploration of the model, Cathedral visits and their research into pilgrims journeys.

\section{CONCLUSION}

Open Virtual Worlds provide a flexible platform offering holistic support to the development and deployment of distributed interactive 3D applications. These are particularly appropriate for developing historic reconstructions related to the promotion of cultural heritage as they facilitate cooperation and collaboration during the development process and provide an immersive environment upon deployment. This allows technical experts and domain scholars to collaborate in the creation of historically accurate environments and allows both physical and intangible aspects of the past to be modelled.

Virtual worlds provide an interactive 3D environment users can explore and shape through the proxy of an avatar. They provide a framework which may be used to develop 3D applications. The framework enables distributed access through virtual world clients and collaborative working enriched through the sense of presence achieved with avatars. Whilst the cost of the service provided by Second Life, the most populace virtual world, limits the scale of reconstructions, the OpenSim server enables individuals and organisations to provide their own service and even to create an interconnected web of reconstructions through a hyper grid. Here it is possible to teleport avatars from place to place much as it is possible to jump around the web using hyper links.

In the 3D space the speed of technological change has led to inverted digital literacies. Children are able to explore 3D spaces using games controllers sometimes before they are able to spell their own name. The ability to configure OVWs in a spectrum of configurations ranging from client server web applications to personalized sandboxes combined with their flexibility in supporting multiple user interfaces including traditional key board and mouse, games controllers and touch free gesture control combine to allow a reconstruction to be deployed in a range of contexts.

\section{REFERENCES}

[1] J. Jacobson, "Ancient architecture in virtual reality; does visual immersion really aid learning?" Ph.D. dissertation, University of Pittsburgh, 2008.

[2] R. Fawcett, The architecture of the Scottish medieval Church. Yale University Press, 2011.

[3] R. Fawcett, J. Luxford, and R. Oram, "Scottish medieval parish churches: the evidence from the diocese of Dunblane and Dunkeld," Antiquaries Journal, vol. 90, 2010.

[4] A. Miller, I. Oliver, and C. Allison, "Demiurge: A toolkit for supporting educational developments in virtual worlds," in 2th Annual Conference, of the Educational Academy Subject Center for Information and Computer Science Belfast., 2011.

[5] L. M. Sequira and L. C. Morgado, "Virtual archeaology in Second Life and OpenSimulator," Journal for Virtual World Research, vol. 6, pp. 118, 2013.

[6] Baba, otakup0pe, jhurliman, J. Malthus, mcortez, D. McLean, L. Coder, A. Zaius, jradford, Lancej, frob, and L. Khalifa, "libopenmetaverse," http://www.openmetaverse.org/projects/libopenmetaverse, [Online; accessed 28-March-2011].

[7] J. McCaffery, A. Miller, and C. Allison, "Extending the use of virtual worlds as an educational platform - network island: An advanced learning environment for teaching internet routing algorithms," in CSEDU (1), 2011, pp. 279-284.

[8] V. M. Lopez-Menchero and A. Grande. The principles of the seville charter. WWW. [Online]. Available: http://cipa.icomos.org/fileadmin/template/doc/PRAGUE/096.pdf

[9] R. Fawcett, S. Foster, and C. J. Tabraham, St Andrews Cathedral. Historic Scotland, 2003. 